\title{
Autisme et douleur - analyse bibliographique
}

\author{
Amandine Dubois PhD, Cécile Rattaz PhD, René Pry Pr, Amaria Baghdadli Pr
}

\begin{abstract}
A Dubois, C Rattaz, R Pry, A Baghdadli. Autisme et douleur analyse bibliographique. Pain Res Manage 2010;15(4):245-253.

La présente analyse bibliographique a pour objectif de réaliser un bilan des travaux publiés dans le champ de la douleur et de l'autisme. L'article aborde, dans un premier temps, les études publiées concernant les modes d'expression de la douleur observés dans cette population. Différentes hypothèses permettant d'expliquer les particularités expressives des personnes avec autisme sont ensuite passées en revue : excès d'endorphines, particularités dans le traitement sensoriel, déficit sociocommunicatif. Cette analyse bibliographique aborde, pour terminer, la question de l'évaluation et de la prise en compte de la douleur chez les personnes avec autisme. Les auteurs concluent à l'absence d'homogénéité des résultats des études publiées et au besoin de poursuivre les recherches afin de parvenir à des données consensuelles sur un domaine d'étude encore peu exploité au plan scientifique. Sur un plan clinique, l'approfondissement des connaissances dans ce domaine devrait permettre de mettre au point des outils d'évaluation de la douleur et d'ainsi en assurer une meilleure prise en charge au quotidien.
\end{abstract}

\section{Autism and pain - a literature review}

\begin{abstract}
The purpose of the present article was to assess the available literature concerning pain and autism. First, authors summarized the published articles on pain reactivity in people with autism. Second, the hypotheses envisaged to explain the presence of expressive particularities in people with autism spectrum disorders were reviewed; these included endogenous opioid excess theory, sensorial abnormalities and sociocommunicative deficit. Finally, the present review dealt with the tools available to assess and manage pain in people with autism. In conclusion, the authors revealed the need for more research to obtain more consensual data and provided some recommendations in this domain that were underexploited by the scientific community. From a clinical point of view, more knowledge about pain in people with autism should enable the development of specific assessment tools and, consequently, better pain management in daily care.
\end{abstract}

Key Words: Assessment; Autism; Expression; Pain; Sensory

Mots-clés : Autisme, douleur, évaluation, expression, sensorialité

L a douleur est un domaine de recherche et d'intérêt croissant depuis une trentaine d'années. Des progrès considérables ont ainsi été réalisés dans le secteur des connaissances du fonctionnement du système nociceptif, mais également de l'évaluation et de la prise en charge de la douleur. Ces avancées ont permis d'écarter de nombreuses idées reçues longtemps persistantes, concernant par exemple l'insensibilité à la douleur des nouveau-nés et, plus récemment, des personnes porteuses d'un handicap mental ou d'un trouble du développement. Aujourd'hui, rares sont les cliniciens et les chercheurs qui défendent l'idée d'une insensibilité à la douleur chez les personnes porteuses d'une déficience intellectuelle ou chez les enfants atteints de troubles autistiques. Les familles et les professionnels éducatifs et soignants affrontent régulièrement les problématiques liées à la douleur dans les situations de la vie quotidienne. Néanmoins, la douleur chez les enfants ayant un trouble du spectre autistique reste un domaine encore peu examiné par la communauté scientifique. Beaucoup de questions persistent et restent parfois non résolues : Ces enfants ressentent-ils la douleur de la même manière que les enfants n'ayant pas de trouble autistique ? Quels sont les facteurs qui jouent un rôle dans l'expérience de la douleur ? Enfin, quels sont les moyens actuels disponibles pour repérer et circonscrire la douleur auprès d'enfants dont la principale difficulté est de communiquer?

La présente analyse bibliographique a pour objectif de réaliser un bilan des travaux publiés jusqu'à présent, d'apporter des éléments de réponse à quelques-unes des interrogations évoquées ci-dessus et de mettre en évidence les points qu'il reste à éclaircir concernant la douleur chez les enfants avec autisme. Les éléments des publications citées dans ce travail sont issus d'une recherche transdisciplinaire menée à partir de différentes bases de données ainsi que de portails d'éditeurs internationaux (Medline, Cairn, Science Direct, PsycINFO) et de mots-clés référencés, tels que « douleur, autisme, expression, évaluation, sensation, déficience intellectuelle, opioïdes » (en anglais pain, autistic disorder, expression, assessment, sensation, developmental disabilities, opioids). Au total, 73 articles et chapitres d'ouvrages, relevant à la fois de travaux empiriques et théoriques publiés depuis les années 1970, sont décrits et synthétisés dans ce travail.

\section{QUE SAIT-ON AUJOURD'HUI DES MODALITÉS D'EXPRESSION DE LA DOULEUR DANS L'AUTISME ?}

La Classification internationale des troubles mentaux (1) ainsi que le Manuel diagnostique et statistique des troubles mentaux (DSM-IV-TR) (2) définissent l'autisme comme un trouble envahissant du développement, dont le taux de prévalence est estimé aux environs de dix personnes sur 10000 (3), caractérisé par l'altération des capacités de communication et des interactions sociales réciproques et par la présence de comportements répétitifs et stéréotypés. L'autisme s'associe souvent à des troubles du langage et à un retard mental qui limitent 
d'autant plus les capacités de communication. Ses formes cliniques sont multiples, raison pour laquelle le terme de troubles du spectre autistique est privilégié à l'heure actuelle.

L'enfant ayant un trouble du spectre autistique peut parfois vivre avec des pathologies spécifiques, chroniques ou aiguës (épilepsie, pathologies gastro-intestinales) (4,5), mais également, comme tout autre enfant, devoir faire face à des interventions de soins dentaires ou médicales et à des accidents de la vie courante pouvant être à l'origine de sensations douloureuses $(6,7)$. L'entourage familial, les éducateurs et les soignants achoppent sur des difficultés quand il s'agit de déceler cette douleur, de la localiser, d'évaluer son intensité et de la traiter. Ces enfants ont, par définition, des difficultés dans le domaine de la communication et des interactions sociales qui concernent tous les aspects de la vie quotidienne, notamment quand il s'agit de transmettre et d'exprimer des états internes émotionnels et douloureux. L'idée d'une insensibilité à la douleur dans l'autisme, ou du moins de la diminution de la sensibilité, est comparable à celle formulée il y a une trentaine d'années au sujet de l'insensibilité à la douleur des bébés et enfants en âge préverbal, qui reposait alors sur l'idée d'une immaturité neurologique et cérébrale. Ce même type de préjugé a été appliqué aux personnes porteuses d'une déficience intellectuelle profonde, décrites par Biersdorff en 1991 (8) comme insensibles ou indifférentes à la douleur afin d'expliquer une apparente absence de réactivité. Concernant précisément l'autisme, l'hypothèse d'une insensibilité à la douleur est née de l'observation, chez certains enfants, de comportements particuliers en réaction à cette sensation. La question est loin de faire l'unanimité parmi les chercheurs. En effet, dès le début des travaux de recherche, des controverses sont apparues entre les études. Certaines ont pu montrer la présence de modalités expressives particulières, d'autres, l'absence de réaction face à une douleur ou, à l'inverse, des expressions exacerbées. L'ensemble des travaux s'accorde néanmoins sur le fait que l'expression de la douleur chez les enfants avec autisme est altérée et caractérisée par la présence de particularités expressives.

Parmi les auteurs ayant abordé ce sujet, Tordjman et coll. $(9,10)$ ont étudié la réactivité à la douleur auprès d'un groupe d'enfants avec autisme comparé à un groupe d'enfants témoins (appariement réalisé selon l'âge, le sexe et le stade pubertaire). Dans ces travaux, les auteurs décrivent les réactions des enfants lors de différentes situations douloureuses d'après la mesure du rythme cardiaque ainsi que du taux d'endorphine (la $\beta$-endorphine) ainsi que la réactivité comportementale au moyen de la PL-BPRS (Pre-Linguistic Behavioral Pain Reactivity Scale). Cette échelle permet de coter la réactivité à la douleur sur une échelle de cinq classes (réactions paradoxales, absence de réactivité, diminution de la réactivité, réactivité normale et hyperréactivité à la douleur). Les résultats montrent tout d'abord une réactivité comportementale à la douleur réduite chez les enfants avec autisme, que ce soit à domicile (réactions évaluées par les parents), en institution (réactions évaluées par deux personnes en charge de l'enfant) ou durant une ponction veineuse (réactions évaluées par un infirmier et un pédopsychiatre). Les auteurs montrent, par ailleurs, la présence de troubles du comportement (automutilation, comportements stéréotypés, conduites agressives envers autrui et retrait autistique) immédiatement après une expérience de douleur.
Quelques plaintes verbales ont été décrites, mais sans localisation précise de la zone douloureuse ni retrait ou protection de la zone du corps touchée. Les auteurs notent également l'existence de réponses neurovégétatives anormalement élevées (tachycardie, augmentation du taux d'endorphine plus élevé que chez les enfants du groupe témoin). Cette dernière observation laisse à penser que l'absence de réactivité comportementale ne signifie pas une absence de perception de la douleur. L'apparente diminution de réactivité envers la douleur relèverait ainsi plutôt d'un mode d'expression comportementale et physiologique particulier. Par ailleurs, l'hyporéactivité notée dans ces études peut s'expliquer par le choix de l'échelle PL-BPRS qui, se fondant sur une description globale des réactions de l'enfant, fait appel à la subjectivité de l'observateur et ne permet probablement pas de mettre en évidence des modes de réaction à la douleur moins habituels ou plus idiosyncrasiques. Enfin, ces auteurs soulignent l'importance de faire la distinction entre les différentes sources de douleur. Il apparaît en effet que, lors de certaines situations douloureuses (brûlure, par exemple), les enfants avec autisme peuvent présenter une réactivité comportementale similaire à celle des enfants du groupe témoin. Parallèlement à ces études, Militerni et coll. (11) ont réalisé des entretiens auprès de parents d'enfants avec autisme afin de repérer les modalités expressives de la douleur. Les indicateurs retenus dans cette étude manquent ici aussi de précision (réactivité normale, faible, très faible). Néanmoins, le témoignage des parents permet de mettre en évidence que les réactions à la douleur de leur enfant sont altérées par rapport à celles d'enfants issus de la population générale. GilbertMacLeod et coll. (12) confirment, lors d'une étude sur des enfants porteurs d'un retard de développement (comprenant des enfants avec autisme), la présence de réactions moins marquées et intenses face à la douleur. Ce sont les premiers auteurs à considérer l'absence de réaction comportementale comme un mode d'expression de la douleur. Quelques années plus tard, Pernon et Rattaz (13) ont vérifié la réactivité à la douleur d'enfants avec autisme. Ils notent une réaction à la stimulation douloureuse se traduisant davantage par des réponses motrices et vocales que faciales. Ceci sous-entend que, contrairement aux enfants issus de la population générale, l'expression faciale n'est pas un marqueur expressif prédominant chez les enfants ayant un trouble du spectre autistique lors d'une sensation douloureuse.

Quelques études viennent néanmoins nuancer toutes ces observations. Nader et coll. (14) ont en effet observé les réactions faciales d'enfants avec autisme lors d'une ponction veineuse comparées à celles d'enfants témoins. Durant le stimulus douloureux, ces auteurs, contrairement aux études précédentes, observent une augmentation des mimiques faciales plus importante que chez les enfants témoins. Les différences de résultats observées comparativement aux précédents travaux proviennent sans doute de l'utilisation, dans cette étude, d'un système de cotation précis des mimiques faciales, en l'occurrence le CFCS (Child Facial Coding System), et non d'une échelle globale telle que la PL-BPRS $(9,10)$. Récemment, Messmer et coll. (15) ont publié une étude qui montre que les mimiques faciales exprimées par des enfants avec autisme lors d'une sensation douloureuse (ponction veineuse) sont un bon indicateur pour estimer l'intensité de la douleur. Les résultats montrent aussi que ces enfants sont capables de communiquer de manière 
spécifique leur expérience de la douleur par l'expression faciale. Les derniers travaux de recherche publiés semblent aller dans ce sens et suggèrent que les enfants ayant un trouble du spectre autistique ont une expression certes particulière, mais spécifique à une situation douloureuse. Enfin, l'autisme s'associe fréquemment à une déficience intellectuelle. Or, les études publiées chez les personnes porteuses d'une déficience intellectuelle ont permis de mettre en évidence la présence de particularités expressives. En effet, des comportements d'auto-agression, des attitudes dites de freezing ou sidération ainsi que la production d'expressions vocales particulières (grognement, grincements de dents, rires), de mimiques faciales singulières (yeux écarquillés, expression de sourire) ou encore l'augmentation de la spasticité, ont pu être décrits dans différents travaux de recherche (16-18).

Au cours du temps, différentes hypothèses ont été émises afin d'expliquer les particularités observées chez les personnes avec autisme : la présence d'un dysfonctionnement du système neurochimique, plus précisément du système opioïde, l'existence de particularités touchant le système sensoriel et enfin, l'implication du niveau de développement dans les capacités à détecter et à communiquer à son environnement proche une expérience douloureuse. Dans les paragraphes qui suivent, nous exposons chacune de ces hypothèses.

\section{TOUT D'ABORD, LE SYSTÈME NEUROCHIMIQUE}

En 1979, sur la base d'expérimentations réalisées auprès d'animaux de laboratoire, Panksepp (19) formule une théorie concernant un excédent d'opioïdes dans l'autisme. Selon lui, certains troubles autistiques, tels que le manque de réactivité, l'absence de partage de la sensation (recherche de réconfort, plaintes) et la présence de comportements restreints et stéréotypés, seraient attribuables à un excédent d'opioïdes endogènes, parmi lesquels les endorphines. Ces dernières agissent notamment sur la labilité émotionnelle, les comportements stéréotypés et la sensibilité à la douleur. L'absence de réactivité observée chez certains enfants avec autisme lors d'une sensation douloureuse a ainsi été mise en lien avec la présence d'une surcharge d'opioïdes qui les rendrait moins sensibles, voire insensibles à la douleur. Des travaux ont montré en effet des taux d'endorphine (en l'occurrence la $\beta$-endorphine) plus élevés dans le sang et le liquide céphalorachidien des enfants avec autisme que dans celui des enfants de groupes témoins (9,20-25). Concernant les données pharmacologiques, à partir des années 1985, une série d'essais thérapeutiques a été effectuée au moyen de traitements ayant un effet antagoniste aux opioïdes (traitement par le naloxone, le naltrexone). Les travaux menés montrent une diminution des symptômes autistiques (comportements d'automutilation, retrait autistique, stéréotypies, hyperactivité) chez des enfants avec autisme ayant reçu un de ces traitements par rapport à des enfants ayant reçu un placebo $(21,23,26,27)$. Néanmoins, la dérégulation du système opioïde n'a jamais fait consensus parmi les travaux. Des chercheurs montrent, en effet, chez des enfants avec autisme, des taux d'opioïdes équivalents (28-31), voire inférieurs (31-35), à ceux des enfants de groupes témoins. Parmi les derniers travaux réalisés dans ce domaine, l'étude de Dettmer et coll. (36) mesurant le taux d'opioïdes dans les urines n'a pas permis de détecter, chez les enfants avec autisme, un taux d'opioïdes supérieur au seuil observé chez des enfants du groupe témoin. Récemment, Tordjman et coll. (10) ont montré un taux plus élevé de $\beta$-endorphines dans le liquide céphalorachidien d'enfants avec autisme que dans celui d'un groupe témoin. Ces auteurs ont approfondi l'analyse et mis en évidence un lien significatif entre la sévérité de l'autisme (évaluée au moyen de l'Autism Diagnostic Interview - Revised), le quotient intellectuel (QI) et le taux d'endorphine. Les enfants ayant un trouble autistique d'intensité sévère et un faible QI ont un taux plus élevé que ceux ayant une intensité légère ou moyenne et un QI plus élevé. Dans les deux cas, le taux d'endorphine reste néanmoins supérieur à celui des enfants du groupe témoin. Enfin, concernant précisément la douleur, les auteurs concluent à une absence de relation entre le taux de $\beta$-endorphine et la qualité de la réactivité envers la douleur, quelle que soit la situation d'observation (à domicile ou lors d'une ponction veineuse). Cette dernière étude, dont les moyens techniques sont plus élaborés et fiables que ceux utilisés dans les années 1980-1990, vient donc remettre en question la théorie élaborée par Panksepp (19) décrivant les enfants avec autisme comme insensibles à la douleur. Par ailleurs, même dans l'hypothèse d'une relation entre certains symptômes autistiques, tels que les stéréotypies ou les automutilations, et un dérèglement du système opioïde, les études ne permettent pas d'établir le sens de cette relation. Concernant précisément les automutilations, ces dernières sont en effet susceptibles de provoquer une décharge d'opioïdes et, parallèlement, une apparente insensibilité à la douleur. Inversement, un excédent d'opiödes peut mener à des comportements auto-agressifs ou à une diminution de réactivité à la douleur. Symons et Danov (37) notent que la nature de la relation entre les automutilations et la douleur n'est ainsi pas clairement identifiée, la douleur pouvant être à la fois la cause et la conséquence des automutilations. Les auteurs ajoutent que la plupart des modèles qui s'intéressent à l'émergence et au maintien des automutilations ne prennent pas en compte les mécanismes spécifiques à la transmission et à la régulation de la douleur, malgré le fait que les automutilations entrainent des dommages tissulaires et des blessures associées. Les travaux effectués spécifiquement sur le lien entre les comportements d'automutilation et la douleur montrent que les enfants porteurs d'une déficience intellectuelle sévère ayant des comportements d'automutilation n'ont pas une réactivité à la douleur réduite (38) et peuvent même avoir des réactions à la douleur amplifiées (39). Breau et coll. (38) suggèrent par ailleurs que les douleurs chroniques peuvent contribuer à la fréquence des comportements d'automutilation et influencer leur localisation. Cela a d'ailleurs été confirmé par Symons et Danov (37) qui ont montré, chez un jeune garçon de six ans, des réactions à la douleur plus élevées dans les périodes durant lesquelles les automutilations étaient les plus fréquentes. Dans une étude portant sur les zones du corps touchées par les automutilations, Symons et Thompson (40) montrent que $80 \%$ des automutilations sont dirigées vers la tête, essentiellement le front, et vers les mains, essentiellement le dos de la main. Ils notent par ailleurs que le tiers des zones du corps concernées sont des zones dont la stimulation produit une analgésie. Les auteurs suggèrent une possible implication des opioïdes dans la localisation des zones corporelles soumises aux automutilations, certains endroits du corps étant susceptibles de provoquer une libération importante et 
immédiate d'opioïdes, de la même façon que la prise d'une drogue. Dès lors, nous pouvons envisager l'hypothèse selon laquelle des douleurs non traitées peuvent être à l'origine de certains comportements d'automutilation, qui provoquent un apaisement par la libération d'endorphines.

Pour de l'information plus approfondie concernant les données neurochimiques chez les enfants et adultes avec autisme, plusieurs analyses bibliographiques exhaustives ont été publiées et peuvent être consultées $(41,42)$. La revue rédigée par Lam et coll., publiée en 2006 (43), est la plus récente. Les travaux réalisés depuis cette date se sont multipliés, mais l'absence de résultats homogènes et consensuels persiste. Il est à noter également que les études précédemment citées sont entachées de problèmes méthodologiques pouvant limiter la portée des résultats (utilisation d'outils d'évaluation non validés, groupe témoin non équivalent au groupe autiste, faible échantillon, etc.). D'autres études adhérant à l'hypothèse d'une insensibilité à la douleur ont fondé leurs résultats sur de simples intuitions et observations cliniques parfois isolées (44). L'absence de réel consensus et de résultats homogènes fait que l'hypothèse d'une sensibilité réduite à la douleur persiste encore dans les années 2000. D'ailleurs, le DSM-IV-TR, publié cette même année, mentionne la présence d'un seuil élevé à la douleur parmi les caractéristiques et troubles associés à l'autisme (2). Cependant, à l'heure actuelle, l'hypothèse d'une insensibilité à la douleur des enfants avec autisme semble être écartée, et les travaux postulent davantage pour la présence de troubles de la sensorialité dans sa globalité.

\section{MAINTENANT, LE SYSTÈME SENSORIEL}

La douleur est un processus complexe dans lequel interagissent de multiples composantes parmi lesquelles des composantes comportementale, émotionnelle et sensorielle (45). Appréhender la douleur dans sa globalité nécessite donc de s'intéresser également aux aspects sensoriels.

Dans les publications, un courant de recherche portant sur l'étude de la sensorialité dans l'autisme se développe depuis quelques années. Rogers et Ozonoff (46) ont réalisé une revue critique des travaux publiés sur le thème des particularités sensorielles observées chez les enfants atteints de troubles du spectre autistique. Au cours de ce travail, ils remarquent que ces particularités ne distinguent pas forcément ces enfants de ceux porteurs d'autres troubles du développement. Néanmoins, ces auteurs notent que les particularités sensorielles sont effectivement plus fréquentes et importantes chez les enfants ayant un trouble du spectre autistique que chez ceux ayant un développement type. L'étude de Pernon et coll. (47) montre que lors d'une sensation provoquée par une température chaude, l'intensité de l'expression faciale des enfants avec autisme est supérieure à celle des enfants du groupe témoin. Ces résultats tendent donc à montrer que les enfants avec autisme semblent avoir une sensibilité accrue face à certains stimuli. Par ailleurs, au cours de leur étude, les auteurs ont également observé des expressions émotionnelles singulières et spécifiques, comme des expressions de sourire au contact de stimuli tactiles jugés a priori peu agréables (rugosité).

Pour évaluer le système sensoriel et perceptif des enfants avec autisme, la plupart des travaux utilisent la version courte de l'échelle Sensory Profile (Short Sensory Profile [SSP]), outil étalonné sur un échantillon issu de la population générale (48).
Cette échelle permet d'évaluer, à partir d'un questionnaire rempli par les parents, entre autres la sensibilité tactile, auditive, olfactive, visuelle ainsi que la sensibilité au goût. Tomchek et Dunn (49) ont utilisé cet outil et ont montré que $95 \%$ des enfants avec autisme de trois à six ans présentent des dysfonctionnements du système sensoriel, notamment le filtrage auditif et les sensations tactiles. En 2003, Rogers et coll. (50) avaient déjà noté ces résultats en montrant un niveau élevé de particularités sensorielles, notamment concernant la sensibilité tactile, auditive et olfactive, par rapport aux enfants issus de la population générale. Récemment, Crane et coll. (51) ont montré que les particularités dans le traitement sensoriel sont également présentes chez des adultes ayant un trouble du spectre autistique.

Les études ayant été menées au moyen d'outils d'évaluation différents du SSP montrent des résultats similaires (52). Par exemple, Leekam et coll. (53), en utilisant le Diagnostic Interview for Social and Communication Disorder, confirment les résultats précédents chez des enfants avec autisme de trois à 11,5 ans et précisent que les particularités sensorielles sont souvent plurimodales et persistent dans le temps malgré l'effet de l'âge et du quotient intellectuel sur certaines particularités. Baranek et coll. (54) proposent un nouvel outil, le Sensory Experiences Questionnaire adapté, pour évaluer les modalités sensorielles chez les jeunes enfants avec autisme (moins de six ans et demi). Les auteurs confirment une prévalence des particularités sensorielles plus élevée chez les enfants avec autisme (69\%) que chez ceux porteurs d'un retard de développement (38\%) et mettent en évidence un lien entre l'importance de ces particularités et l'âge de développement des enfants : plus ce dernier est bas, plus les particularités sensorielles sont importantes.

Si de nombreuses études ont montré des capacités supérieures chez les personnes avec autisme concernant la discrimination visuelle et auditive (55), les résultats concernant la modalité tactile sont plus contrastés. En effet, des particularités ont été relevées dans des études établies à partir d'hétéroobservations (fournies au moyen de l'échelle SSP) et dans des recueils autobiographiques (56), mais les études psychophysiques qui ont pour objectif d'étudier les seuils de réactivité à des stimuli tactiles ou vibratoires et permettant de fournir des données objectives, sont encore rares. Parmi les études publiées, celle de O'Riordan et Passetti (57) a montré l'absence de différence entre des enfants ayant un autisme de haut niveau et un groupe témoin dans la capacité à discriminer la rugosité de différents types de papier de verre et à détecter des stimuli tactiles sur le bras. Chez des adultes porteurs du syndrome d'Asperger, Blakemore et coll. (58) ont mis en évidence, quant à eux, une hypersensibilité tactile (soit un seuil de détection plus bas) mais dépendante du type de stimulus. En l'occurrence, les auteurs précisent que l'hypersensibilité est observée avec des stimuli vibratoires de haute fréquence $(200 \mathrm{~Hz})$ et non avec des stimuli de basse fréquence $(30 \mathrm{~Hz})$. Enfin, Cascio et coll. (59) ont montré un niveau de perception tactile pour une sensation thermique chaude similaire entre des adultes avec un autisme de haut niveau et des adultes témoins. Ils notent cependant une sensibilité à la stimulation vibratoire et à la douleur thermique plus élevée - soit un seuil de tolérance plus faible - chez les adultes avec autisme. À noter que ces études portent essentiellement sur des personnes sans déficience intellectuelle, 
ayant des compétences verbales suffisamment élaborées pour pouvoir indiquer et préciser leur sensation.

La question des particularités sensorielles dans l'autisme se pose à la vue de nombreux travaux qui mettent l'accent sur des comportements d'hyporéactivité ou d'hyperréactivité à certaines stimulations sensorielles, suggérant la présence de particularités sur le plan quantitatif (notion de seuil) et qualitatif (recherche ou évitement de certaines sensations). Cette question se pose également par l'observation de comportements répétitifs, tels que les stéréotypies. Ces dernières peuvent provoquer, en effet, des sensations tant internes (par exemple, des sensations vestibulaires provoquées par les tournoiements, des sensations musculaires provoquées par l'hypertonie), qu'externes (des sensations visuelles provoquées par le passage d'un objet devant les yeux, des sensations tactiles provoquées par le contact avec certaines surfaces, etc.). Ces différentes observations rendent légitime, par conséquent, le fait de penser que la sensation nociceptive, qui met en jeu le système sensoriel, soit susceptible de présenter des particularités.

\section{POUR FINIR, LES ASPECTS DÉVELOPPEMENTAUX}

Au-delà des aspects neurochimiques et sensoriels, la douleur comporte une composante cognitive, impliquée à la fois dans la capacité à déceler la douleur et sa source et dans la capacité à communiquer cette sensation à son environnement social.

Les travaux publiés dans le domaine de la douleur concernant les personnes porteuses d'une déficience intellectuelle ont permis de mettre en lien le niveau de développement avec la qualité des comportements observés lors d'une douleur. Fanurik et coll. (60) ont suggéré un tel lien en distinguant les enfants selon leur degré de déficience intellectuelle et selon la qualité de leur expression douloureuse. Cette description a d'ailleurs été reprise et confirmée quelques années plus tard (61). Ces auteurs ont qualifié l'expression des enfants porteurs d'une déficience intellectuelle légère à moyenne par le terme d'expression « directe », dans le sens où ces enfants s'expriment principalement par la localisation de la zone douloureuse et la verbalisation avec ou sans localisation, alors que celle des enfants porteurs d'une déficience intellectuelle grave à profonde est qualifiée d' " indirecte », dans le sens où ils s'expriment par des pleurs, des mimiques faciales, des comportements d'autoagression, etc. Hadden et von Baeyer (62) distinguent, quant à eux, les enfants porteurs d'une déficience intellectuelle au regard de leurs compétences en communication expressive. Ils relèvent des différences d'expression entre les enfants capables de verbaliser la douleur et ceux qui ne sont pas en mesure de le faire. Les premiers présentent une expression comportementale commune à celle d'enfants issus de la population générale (mouvements vers la zone douloureuse, gémissements, agitation, etc.), alors que les seconds présentent des particularités expressives (rires, comportements d'auto-agression, langue tirée, etc.). Dubois et coll. (63) confirment la présence d'une hétérogénéité d'expression parmi les enfants porteurs d'une déficience intellectuelle et l'influence du niveau de compétences en communication expressive et socialisation. Les enfants porteurs d'une déficience intellectuelle capables de verbaliser la douleur présentent une expression de la douleur normée qui s'apparente à celle d'enfants de même âge de développement, alors que les enfants qui ne sont pas capables de verbaliser la douleur et qui possèdent un faible niveau en communication et socialisation présentent une expression singulière, qui se caractérise par un manque d'expressivité faciale, vocale ou motrice et par l'exacerbation des comportements autostimulants. L'importance du niveau des compétences développementales ne fait néanmoins pas l'unanimité et est nuancée par quelques auteurs, tels que Zabalia (64), qui estime que l'expérience de vie, exprimée par les expériences sensorielles et affectives vécues par l'enfant, influe principalement sur l'expérience et l'expression douloureuse.

Concernant les études portant spécifiquement sur l'autisme, Tordjman et coll. $(9,10)$ font l'hypothèse que les particularités observées lors d'une situation douloureuse proviendraient d'un trouble de la communication générale (verbale et non verbale) et également de difficultés à reconnaître la sensation ellemême. En effet, les enfants avec autisme semblent capables de percevoir et d'exprimer effectivement un état de mal-être, repérable par exemple par l'expression de pleurs, mais il semble qu'ils aient des difficultés à détecter et à discriminer la sensation de douleur d'autres sensations désagréables. Ces difficultés se traduisent par l'absence de mise en ouvre d'actions efficaces pour échapper à la douleur et y faire face (retrait, protection, etc.). Gilbert-MacLeod et coll. (12) émettent, quant à eux, une hypothèse unique en termes de déficit sociocommunicatif. Selon ces auteurs, les réponses altérées à la douleur peuvent être interprétées comme le résultat de déficits de certains comportements sociaux (pointer, avoir une attention conjointe, vocaliser, etc.). Nader et coll. (14) ont repris cette hypothèse afin d'expliquer l'augmentation de l'activité faciale observée chez les enfants avec autisme. Ce résultat est interprété comme un manque d'inhibition, en lien avec une incapacité à réguler l'expression comportementale lors d'émotions à valence négative.

Ces différents travaux permettent donc de mettre en évidence l'effet du niveau de développement dans l'expression de la douleur, en particulier sur le plan des compétences sociocommunicatives.

\section{EXPRESSION DE LA DOULEUR PARTICULIÈRE DANS L'AUTISME : VERS UN MODÈLE INTÉGRÉ} $\mathrm{Au}$ regard des différentes hypothèses émises dans le but d'expliquer l'altération de la qualité de l'expression de la douleur, il semble difficile de privilégier une hypothèse aux dépens d'une autre. Comme le suggèrent Hadjistavropoulos et Craig (65), l'expression de la douleur peut être conceptualisée comme un modèle de communication du type «Émetteur $(\mathrm{A}) \rightarrow$ Message (B) $\rightarrow$ Récepteur (C) », dans lequel interviennent (A) la sensation interne ressentie par la personne et modulée par des facteurs contextuels et personnels; (B) le message, à savoir les comportements exprimés par cette personne, qui peut dépendre de processus volontaires (expression verbale) ou automatiques (comportements non verbaux); et (C) l'observateur, responsable de l'évaluation et de la prise en charge de la douleur, dont la qualité du décodage va dépendre de la clarté du message et d'éléments personnels.

Ce modèle, appliqué à la question de la douleur chez les personnes avec autisme, permet de conceptualiser les différents facteurs susceptibles d'entrer en jeu ainsi que les différents niveaux qui peuvent être perturbés (figure 1). Concernant la personne avec autisme, la sensation ressentie 


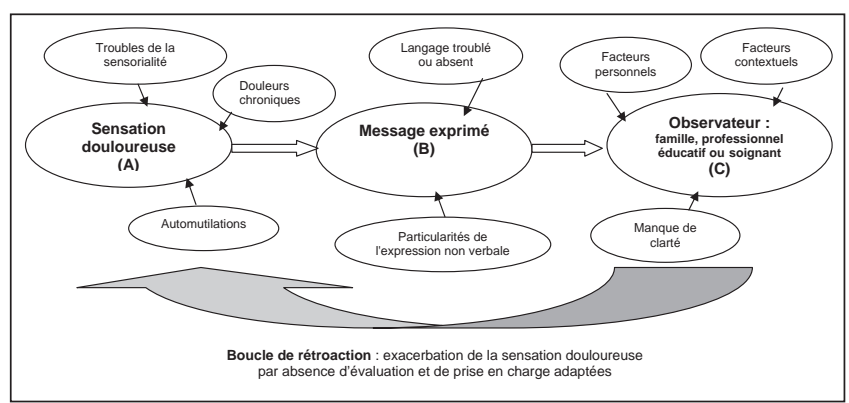

Figure 1) Expression de la douleur chez les personnes ayant un trouble du spectre autistique : modèle intégré, inspiré du modèle de Hadjistavropoulos et Craig (65). A Sensation douloureuse, dont la qualité peut être altérée chez des personnes avec autisme à cause de troubles de la sensorialité, de douleurs chroniques récurrentes ou de comportements d'automutilation. B Message exprimé, altéré par un langage verbal troublé et des particularités concernant l'expression non verbale (regard, attention, etc.). C Observateur : famille, professionnel éducatif ou soignant, pour qui l'évaluation et la détection de la douleur peuvent être rendues difficiles à cause de facteurs personnels et contextuels, et par manque de clarté de l'expression comportementale. Boucle de rétroaction entre l'observateur et la sensation douloureuse : une mauvaise évaluation, détection et prise en charge de la douleur par l'observateur peuvent entraîner une exacerbation de la douleur et, le cas échéant, du trouble somatique sous-jacent

(A) peut être altérée pour plusieurs raisons. Il existe d'abord des particularités sensorielles, décrites tant par les personnes autistes elles-mêmes (56) que par l'entourage dans des questionnaires spécifiques tels que le SSP (cf. plus haut). Il peut également exister des douleurs qui, insuffisamment prises en charge, se chronicisent, avec pour effet une diminution des réactions au fil du temps (phénomène d'habituation), comme cela a été montré par exemple chez des nouveau-nés prématurés qui subissent des interventions douloureuses répétées (66). Enfin, certains troubles du comportement, tels que les automutilations, sont susceptibles de provoquer des douleurs, voire à l'inverse une diminution du seuil de perception de la douleur par le jeu des endorphines. Ensuite, le message exprimé par la personne (B) peut manquer de clarté en raison du déficit sociocommunicatif qui touche à la fois les aspects verbaux (capacité à exprimer ses sensations au moyen du langage) et non verbaux (émission de comportements qui peuvent être décodés par l'observateur). Enfin, concernant le dernier point (C), l'observateur est souvent en difficulté pour décoder le message transmis. De multiples facteurs sont en effet susceptibles d'influencer l'évaluation que peut faire l'entourage de l'enfant. Ces facteurs peuvent être personnels (méconnaissance des modalités d'expression de la douleur dans cette population, impact de sa propre subjectivité dans l'évaluation de la douleur chez autrui), contextuels (idées préconçues quantà l'insensibilité à la douleur chez les personnes porteuses d'un handicap), mais aussi liés au manque de clarté du message, le lien entre le comportement émis et la douleur n'étant pas évident a priori (par exemple une personne qui se frappe la tête parce qu'elle a des douleurs dentaires). Un dernier élément doit enfin être intégré à ce modèle : une boucle de rétroaction entre l'observateur (C) et la sensation ressentie (A). En effet, un message mal transmis par l'émetteur ou mal décodé par l'observateur peut avoir des répercussions sur la qualité de l'évaluation de la douleur (évaluation de son intensité, localisation de la zone douloureuse, etc.) et, par là même, sur la qualité de sa prise en charge (traitement antalgique inefficace ou insuffisant). Cette absence de prise en compte de la douleur efficace peut alors avoir pour conséquence une exacerbation de la sensation douloureuse et une amplification de la pathologie somatique sousjacente. À titre illustratif, pour reprendre l'exemple ci-dessus, une douleur dentaire non décelée amène à une absence de soins adaptés et, par conséquent, à une augmentation de la sensation douloureuse de départ et à une aggravation du problème dentaire.

Ce modèle intégré, qui décrit des dysfonctionnements multiples sur les plans sensoriel, cognitif et comportemental de la douleur, peut donc permettre d'expliquer les particularités expressives observées lors d'une douleur et de proposer une évaluation et une prise en charge qui tiennent compte de ces différents plans.

\section{COMMENT DÉTECTER ET PRENDRE EN COMPTE LA DOULEUR CHEZ LES PERSONNES AYANT UN TROUBLE DU SPECTRE AUTISTIQUE?}

Pour déterminer la douleur, lorsque cela est possible, il est important de s'appuyer sur les parents ou les proches, qui connaissent les comportements habituels de leur enfant et sont les mieux placés pour détecter des modifications comportementales et une éventuelle présence de douleur. Herr et coll. (67) recommandent d'ailleurs vivement leur présence et leur participation lors de l'évaluation de la douleur d'un enfant incapable de reporter verbalement sa douleur. Cependant, concernant les enfants avec autisme, l'évaluation de la douleur semble parfois difficile, y compris pour l'entourage familier. Nader et coll. (14) montrent en effet l'absence de concordance entre l'évaluation de l'expression faciale de l'enfant réalisée par un observateur (à partir de l'échelle CFCS) et l'estimation de l'intensité de la douleur par les parents (à partir de l'échelle des visages classique). Par ailleurs, ils montrent l'absence de lien entre l'évaluation comportementale de la douleur rétrospective des parents (à l'aide de l'échelle NonCommunicating Children's Pain Checklist - Revised [NCCPC-R]) et les comportements observés durant une situation douloureuse. Les enfants les plus réactifs lors de la situation douloureuse sont ceux qui ont été décrits comme les moins sensibles et réactifs à la douleur par les parents. Pour expliquer ces résultats, les auteurs postulent que l'expression de la douleur diffère selon l'environnement (maison, hôpital) et le type de douleur (douleur liée aux soins, douleur quotidienne). Alors qu'ils exprimeraient des comportements atypiques dans une situation de la vie quotidienne, ils s'exprimeraient de manière commune lors d'une situation de soins. Au regard de ces résultats, il est important de pouvoir s'appuyer sur des échelles ou des outils validés qui permettent d'objectiver les réactions de l'enfant. À l'heure actuelle, aucune étude, aucun outil d'évaluation de la douleur n'a été validé et mis à l'essai spécifiquement auprès des enfants et adultes avec autisme. Il existe cependant des outils qui ont été validés auprès d'enfants ou d'adultes porteurs d'une déficience intellectuelle, tels que l'échelle NCCPC-R (16,68), Faces, Legs, Activity, Cry and Consolability - Revised (FLACC-R) (18), Douleur Enfant San 
Salvador (69) ou encore Paediatric Pain Profile (70) (pour une analyse bibliographique des outils validés pour cette population, l'article de Symons et coll. [71] peut être consulté). Certains de ces outils ont été éprouvés auprès d'enfants avec autisme (14), et ces travaux mériteraient d'être poursuivis afin de confirmer les résultats acquis.

La prise en charge de la douleur est entièrement liée aux capacités des parents ou des professionnels à déterminer la source de douleur, à évaluer son intensité et à émettre des hypothèses sur ses origines. Chez une personne qui communique de façon verbale, cela ne pose habituellement pas de problème, et le diagnostic des professionnels peut être réalisé sur la base uniquement d'un entretien relativement poussé avec le patient. Il en est autrement chez des personnes qui ne peuvent communiquer au moyen du langage. Pour reprendre le modèle proposé de Hadjistavropoulos et Craig (65), lorsque le message n'est pas émis de façon claire et ne peut être décodé par le partenaire, il arrive fréquemment que la sensation douloureuse s'exprime d'une autre façon, notamment par des modifications comportementales, voire des troubles du comportement. Pour pallier cette difficulté, une approche intéressante est celle que propose l'analyse fonctionnelle (72), réalisée au moyen d'une grille d'observation très fine du comportement sur une période donnée et qui peut être utilisée par l'entourage pour repérer les modifications comportementales chez un enfant. Cette technique peut permettre de formuler des hypothèses et de détecter la présence d'éventuelles douleurs non identifiées et non traitées pouvant être sources de troubles du comportement. Elle peut également permettre, grâce à la recherche de corrélations entre plusieurs événements, la mise en évidence de liens entre les réactions à la douleur et certains comportements d'automutilation $(37,39,73)$. L'analyse fonctionnelle ne peut cependant être réalisée que pour une personne donnée dans un contexte donné, en réponse à des situations critiques où les douleurs et les problèmes de santé se sont souvent chronicisés. Il importe, par conséquent, de poursuivre les recherches sur les modalités d'expression de la douleur et d'élaborer des outils pouvant être utilisés par des professionnels de première ligne. Si la prise en compte de la douleur en est améliorée, les situations critiques dans lesquelles les trois niveaux du modèle ci-dessus sont simultanément altérés devraient, par conséquent, être exceptionnelles.

\section{RÉFÉRENCES}

1. Organisation mondiale de la santé. CIM 10, Classification statistique internationale des maladies et des problèmes de santé connexes dixième révision. Genève: Organisation mondiale de la santé, 1993.

2. American Psychiatric Association. Diagnostic and Statistical Manual of Mental Disorders IV - Text Revised. Washington: American Psychiatric Association, 2000.

3. Fombonne E. Epidemiological surveys of autism and other pervasive developmental disorders: An update. J Autism Dev Disord 2003;33:365-82.

4. Oberlander TF, Symons FJ. Pain in children and adults with developmental disabilities. Baltimore: Paul H Brookes Publishing, 2006.

5. Gillberg C, Coleman M. Autism and medical disorders: A review of the literature. Dev Med Child Neurol 1996;38:191-202.

6. Lee L, Harrington RA, Chang JJ, Connors SL. Increased risk of injury in children with developmental disabilities. Res Dev Disabil 2008;29:247-55.

7. Breau LM, Camfield CS, McGrath PJ, Finley GA. The incidence of pain in children with severe cognitive impairments. Arch Pediatr Adolesc Med 2003;157:1219-26.

\section{CONCLUSION}

Le niveau de connaissances que nous avons sur la douleur chez les enfants ayant un trouble du spectre autistique est similaire à celui que nous avions il y a quelques années sur les bébés et personnes porteuses d'une déficience intellectuelle. Les raisons de l'absence de recherches dans ce domaine sont sans doute liées à la difficulté de recrutement d'une telle population, à des considérations éthiques, mais également aux idées reçues longtemps persistantes quant aux capacités d'évolution cognitive et psychologique de ces enfants et à la prise de conscience tardive de leur capacité à ressentir et exprimer une douleur.

La présente analyse bibliographique nous a montré l'absence de données consensuelles sur la douleur chez l'enfant avec autisme. Il existe une forte variabilité interindividuelle concernant les modalités d'expression de la douleur, qui peut être probablement mise en lien avec la variabilité des tableaux cliniques regroupés dans la catégorie de troubles du spectre autistique. Par ailleurs, l'absence de consensus peut être expliquée par le manque d'homogénéité dans les méthodes de travail et les outils utilisés. Concernant la description des comportements après une sensation douloureuse, il est important de ne pas s'arrêter à la description des mimiques faciales observées, mais d'évaluer également les réponses comportementales et physiologiques dans leur globalité. À l'avenir, il sera intéressant de mettre au point des outils prenant en compte les spécificités de l'expression de la douleur chez les personnes avec autisme. Il est en effet probable que des comportements qui ne sont pas liés à l'expression de la douleur dans la population générale puissent l'être chez les personnes avec autisme, rendant alors la détection de l'expérience douloureuse difficile pour un observateur exté-rieur faisant appel à son vécu et son expérience propre.

Enfin, la prise en charge de la douleur ne peut se faire de façon efficace sans une évaluation précise et fine de la sensation douloureuse, d'où l'importance de s'appuyer à la fois sur les éléments apportés par les parents, les observations des professionnels qui suivent l'enfant et des outils tels que des grilles d'observation du comportement. Mises en commun, ces différentes sources d'observation peuvent permettre de formuler des hypothèses sur l'origine de la douleur et, par conséquent, de proposer des traitements ou des adaptations de l'environnement afin de la soulager.

8. Biersdorff KK. Pain insensitivity and indifference: Alternative explanations for some medical catastrophes. Ment Retard 1991;29:359-62.

9. Tordjman S, Antoine C, Cohen DJ et coll. Study of the relationships between self-injurious behavior and pain reactivity in infantile autism. Encephale 1999;25:122-34.

10. Tordjman S, Anderson GM, Botbol M et coll. Pain reactivity and plasma $\beta$-endorphin in children and adolescents with autistic disorder. PLoS ONE 2009;4:e5.

11. Militerni R, Bravaccio C, Falco C, Puglisi-Allegra S, Pascucci T, Fico C. Pain reactivity in children with autistic disorder. J Headache Pain 2000;1:53-6.

12. Gilbert-MacLeod CA, Craig KD, Rocha EM, Mathias MD. Everyday pain responses in children with and without developmental delays. J Pediatr Psychol 2000;25:301-8.

13. Pernon E, Rattaz C. Les modes d'expression de la douleur chez l'enfant autiste : Étude comparée. Devenir 2003; 15:263-77.

14. Nader R, Oberlander TF, Chambers CT, Craig KD. Expression of pain in children with autism. Clin J Pain 2004;20:88-97. 
15. Messmer RL, Nader R, Craig KD. Brief report: Judging pain intensity in children with autism undergoing venepuncture: The influence of facial activity. J Autism Dev Disord 2008;38:1391-4.

16. Breau LM, Finley GA, McGrath PJ, Camfield CS. Validation of the non-communicating children's pain checklist - postoperative version. Anesthesiology 2002;96:528-35.

17. Defrin R, Lotan M, Pick CG. The evaluation of acute pain in individuals with cognitive impairment: A differential effect of the level of impairment. Pain 2006;124:312-20.

18. Malviya S, Voepel-Lewis T, Burke C, Merkel S, Tait AR. The revised FLACC observational pain tool: Improved reliabillity and validity for pain assessment in children with cognitive impairment. Paediatr Anaesth 2006;16:258-65.

19. Panksepp J. A neurochemical theory of autism. Trends Neurosci 1979;2:174-7.

20. Bouvard MP, Leboyer M, Launay JM et coll. Low-dose naltrexone effects on plasma chemistries and clinical symptoms in autism: A double-blind, placebo-controlled study. Psychiatry Res 1995;58:191-201.

21. Cazzullo AG, Musetti MC, Musetti L, Bajo S, Sacerdote P, Panerai A. Beta-endorphin levels in peripheral blood mononuclear cells and long-term naltrexone treatment in autistic children. Eur Neuropsychopharmacol 1999;9:361-6.

22. Gillberg C, Terenius L, Lönnerholm G. Endorphin activity in childhood psychosis. Spinal fluid levels in 24 cases. Arch Gen Psychiatry 1985;42:780-3.

23. Leboyer M, Bouvard MP, Launay JM et coll. Brief report: A double-blind study of naltrexone in infantile autism. J Autism Dev Disord 1992;22:309-19.

24. Ross DL, Klykylo WM, Hitzemann R. Reduction of elevated CSF betaendorphin by fenfluramine in infantile autism. Pediatr Neurol 1987;3:83-6.

25. Sandman CA. The opiate hypothesis in autism and self-injury. J Child Adolesc Psychopharmacol 1991;1:237-48.

26. Sandman CA. Beta-endorphin dysregulation in autistic and self-injurious behavior: A neurodevelopmental hypothesis. Synapse 1988;2:193-9.

27. Campbell M, Anderson LT, Small AM, Adams P, Gonzalez NM, Ernst M. Naltrexone in autistic children: Behavioral symptoms and attentional learning. J Am Acad Child Adolesc Psychiatry 1993;32:1283-91.

28. Herman BH, Hammock MK, Arthur-Smith A et coll. Role of opioid peptides in autism: Effects of acute administration of naltrexone. Soc Neurosci 1986;12:1172. (résumé)

29. Ernst M, Devi L, Silva RR et coll. Plasma betaendorphin levels, naltrexone, and haloperidol in autistic children. Psychopharmacol Bull 1993;29:221-7.

30. Hunter LC, O'Hare A, Herron WJ, Fisher LA, Jones GE. Opioid peptides and dipeptidyl peptidase in autism. Dev Med Child 2003;45:121-8.

31. Nagamitsu S, Matsuishi T, Kisa T et coll. CSF $\beta$-endorphin levels in patients with infantile autism. J Autism Dev Disord 1997;27:155-63.

32. Sandman CA, Barron JL, Chiez-DeMet A, DeMet EM. Brief report: Plasma $\beta$-endorphin and cortisol levels in autistic patients. J Autism Dev Disord 1991;21:83-7.

33. Gillberg C, Hagberg B, Witt-Engerstöm I, Eriksson I. CSF beta-endorphins in childhood neuropsychiatric disorders. Brain Dev 1990;12:88-92.

34. Weizman R, Weizman A, Tyano S, Szekely BA, Sarne YH. Humoralendorphin blood levels in autism in autistic, schizophrenic and healthy subjects. Psychopharmacologia 1984;82:363-70.

35. Weizman R, Gil-Ad I, Dick J, Tyano S, Szekely G, Laron Z. Low plasma immunoreactive beta-endorphin. J Am Acad Child Adolesc Psychiatry 1988;27:430-33.

36. Dettmer K, Hanna D, Whetstone P, Hansen R, Hammock BD. Autism and urinary exogenous neuropeptides: Development of an on-line SPE-HPLC-tandem mass spectrometry method to test the opioid excess theory. Anal Bioanal Chem 2007;388:1643-51

37. Symons FJ, Danov SE. A prospective clinical analysis of pain behavior and self-injurious behavior. Pain 2005;117:473-7.

38. Breau LM, Camfield CS, Symons FJ et coll. Relation between pain and self-injurious behavior in non-verbal children with severe cognitive impairments. J Pediatr 2003;142:498-503.

39. Symons FJ, Harper VN, McGrath PJ, Breau LM, Bodfish JW. Evidence of increased non-verbal behavioral signs of pain in adults with neurodevelopmental disorders and chronic self-injury. Res Dev Disabil 2009;30:521-8

40. Symons FJ, Thompson T. Self-injurious behaviour and body site preference. J Intellect Disabil Res 1997;41:456-68.

41. Gillberg C. Endogeneous opioids and opiate antagonists in autism: Brief review of empirical findings and implications for clinicians. Dev Med Child Neurol 1995;37:239-45.

42. Shattock P, Whiteley P. Biochemical aspects in autism spectrum disorders: Updating the opioid-excess theory and presenting new opportunities for biomedical intervention. Expert Opin Ther Targets 2002;6:175-83.

43. Lam KS, Aman MG, Arnold LE. Neurochemical correlates of autistic disorder: A review of the literature. Res Dev Disabil 2006;27:254-89.

44. Rutherford MD. A retrospective journal-based case study of an infant with autism and his twin. Neurocase 2005;11:129-37.

45. Merskey H, Bogduk N. Classification of chronic pain. Descriptions of chronic pain syndromes and definitions of pain terms. Seattle: IASP Press, 1994.

46. Rogers SJ, Ozonoff S. Annotation: What do we know about sensory dysfunction in autism? A critical review of the empirical evidence. J Child Psychol Psychiatry 2005;46:1255-68.

47. Pernon E, Pry R, Baghdadli A. Autism: Tactile perception and emotion. J Intellect Disabil Res 2007;51:580-7.

48. Dunn W, Westman K. The sensory profile: The performance of a national sample of children without disabilities. Am J Occup Ther 1997;51:25-34.

49. Tomchek SD, Dunn W. Sensory processing in children with and without autism: A comparative study using the short sensory profile. Am J Occup Ther 2007;61:190-200.

50. Rogers SJ, Hepburn S, Wehner E. Parent reports of sensory symptoms in toddlers with autism and those with other developmental disorders. J Autism Dev Disord 2003;33:631-42.

51. Crane L, Goddard L, Pring L. Sensory processing in adults with autism spectrum disorders. Autism 2009;13:215-28.

52. Ben-Sasson A, Hen L, Fluss R, Cermak SA, Engel-Yeger B, Gal E. A meta-analysis of sensory modulation symptoms in individuals with autism spectrum disorders. J Autism Dev Disord 2009;39:1-11.

53. Leekam SR, Nieto C, Libby SJ, Wing L, Gould J. Describing the sensory abnormalities of children and adults with autism. J Autism Dev Disord 2007;37:894-910.

54. Baranek GT, David FJ, Poe MD, Stone WL, Watson LR. Sensory experiences questionnaire: Discriminating sensory features in young children with autism, developmental delays, and typical development. J Child Psychol Psychiatry 2006;47:591-601.

55. Mottron L, Burack J. Enhanced perceptual functioning in the development of autism. Dans: Burack JA, Charman T, Yirmiya N, Zelazo PR, éd. The development of autism: Perspectives from theory and research. Mahwah: Erlbaum, 2001:131-48.

56. Grandin T. Emergence: Labeled Autistic. Novato: Arena Press, 1986

57. O'Riordan M, Passetti F. Discrimination in autism within different sensory modalities. J Autism Dev Disord 2006;36:665-75.

58. Blakemore SJ, Tavassoli T, Calò S et coll. Tactile sensitivity in Asperger syndrome. Brain Cogn 2006;61:5-13.

59. Cascio C, McGlone F, Folger S et coll. Tactile perception in adults with autism: A multidimensional psychophysical study. J Autism Dev Disord 2008;38:127-37.

60. Fanurik D, Koh JL, Schmitz ML, Harrison RD, Conrad TM. Children with cognitive impairment: Parent report of pain and coping. J Dev Behav Pediatr 1999;20:228-34.

61. Voepel-Lewis T, Malviya S, Tait AR. Validity of parent ratings as proxy measures of pain in children with cognitive impairment. Pain Manag Nurs 2005;6:168-74.

62. Hadden KL, von Baeyer CL. Pain in children with cerebral palsy: Common triggers and expressive behaviors. Pain 2002;99:281-8.

63. Dubois A, Capdevila X, Bringuier S, Pry R. Pain in children with an intellectual disability. Eur J Pain 2010. (Sous presse)

64. Zabalia M. Pour une psychologie de l'enfant face à la douleur. Enfance 2006;1:5-19.

65. Hadjistavropoulos T, Craig KD. A theorical framework for understanding self-report and observational measures of pain: A communications model. Behav Res Ther 2002;40:551-70.

66. Johnston C, Stevens B. Experience in a neonatal intensive care unit affects pain response. Pediatrics 1996;98:925-30. 
67. Herr K, Coyne PJ, Key T et coll. Pain assessment in the nonverbal patient: Position statement with clinical practice recommendations. Pain Manag Nurs 2006;7:44-52.

68. Breau LM, McGrath PJ, Camfield CS, Finley GA. Psychometric properties of the non-communicating children's pain checklist-revised. Pain 2002;99:349-57.

69. Collignon P, Giusiano B. Validation of a pain evaluation scale for patients with cerebral palsy. Eur J Pain 2001;5:433-42.

70. Hunt A, Goldman A, Seers K et coll. Clinical validation of the Paediatric Pain Profile. Dev Med Child Neurol 2004:46:9-18.
71. Symons FJ, Shinde SK, Gilles E. Perspectives on pain and intellectual disability. J Intellect Disabil Res 2008;52:275-86.

72. O'Neill RE, Horner RH, Sprague JR, Albin RW. Évaluation fonctionnelle et développement de programmes d'assistance pour les comportements problématiques : manuel pratique. Paris: De Boeck Université, 2008.

73. Bosch J, Van Dyke DC, Smith SM, Poulton S. Role of medical conditions in the exacerbation of self-injurious behavior: An exploratory study. Ment Retard 1997;35:124-30. 


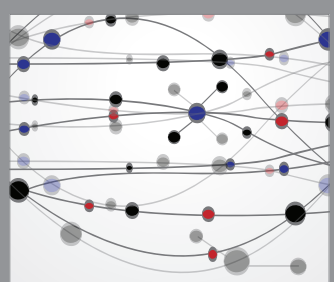

The Scientific World Journal
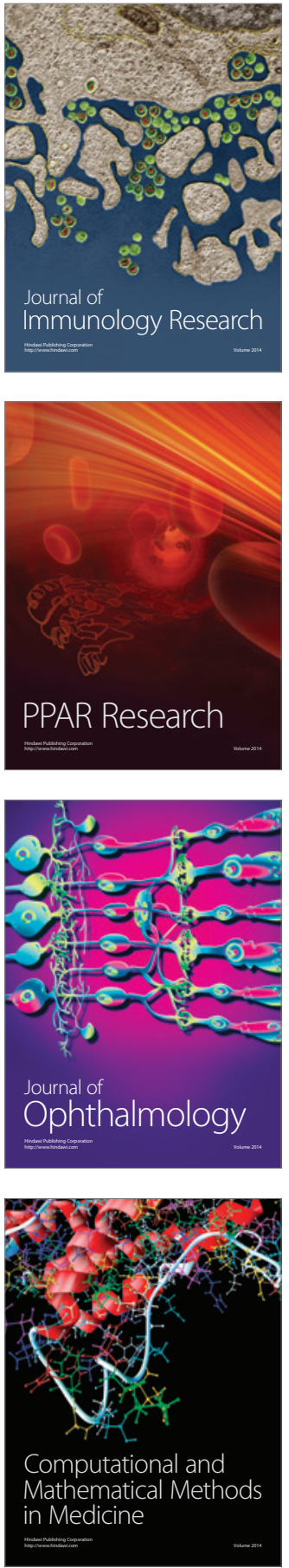

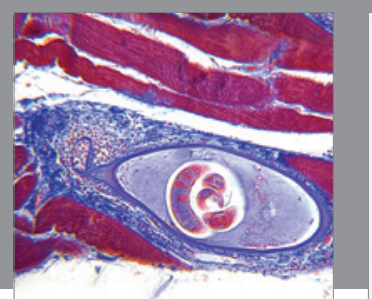

Gastroenterology Research and Practice

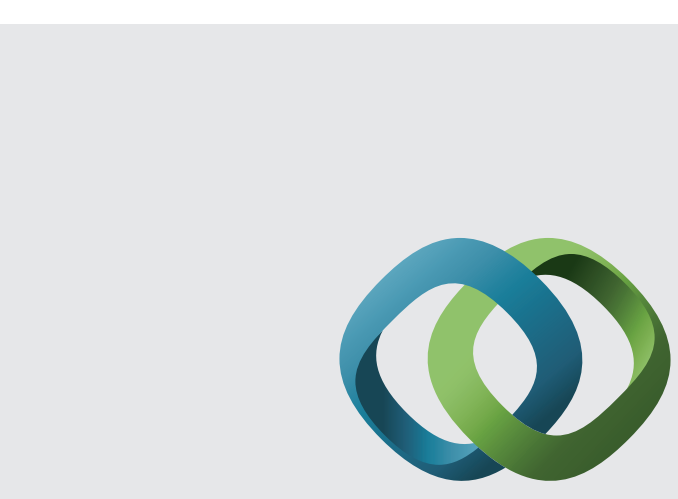

\section{Hindawi}

Submit your manuscripts at

http://www.hindawi.com
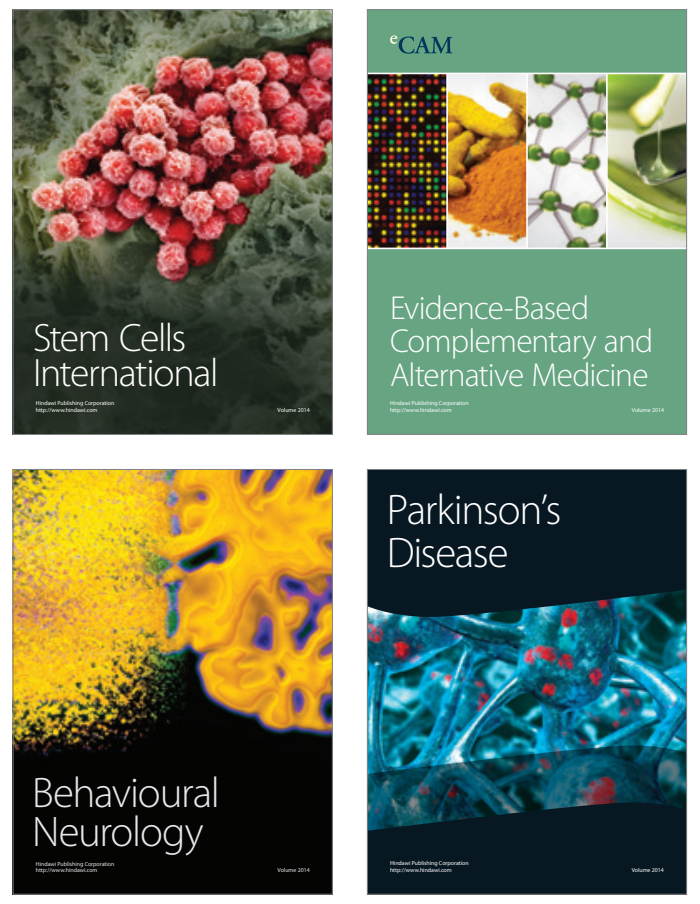
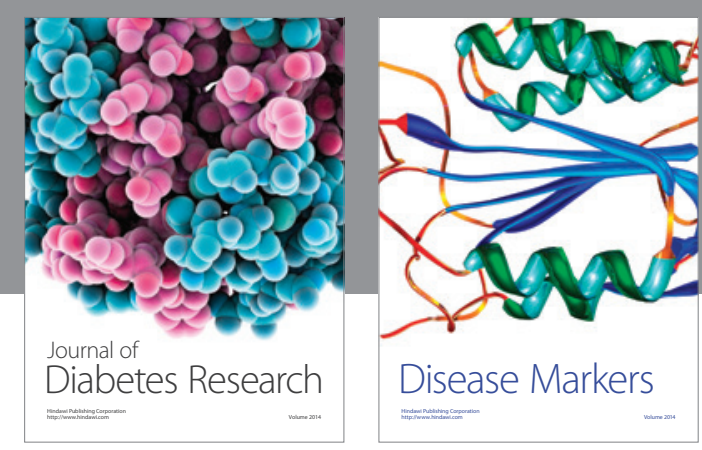

Disease Markers
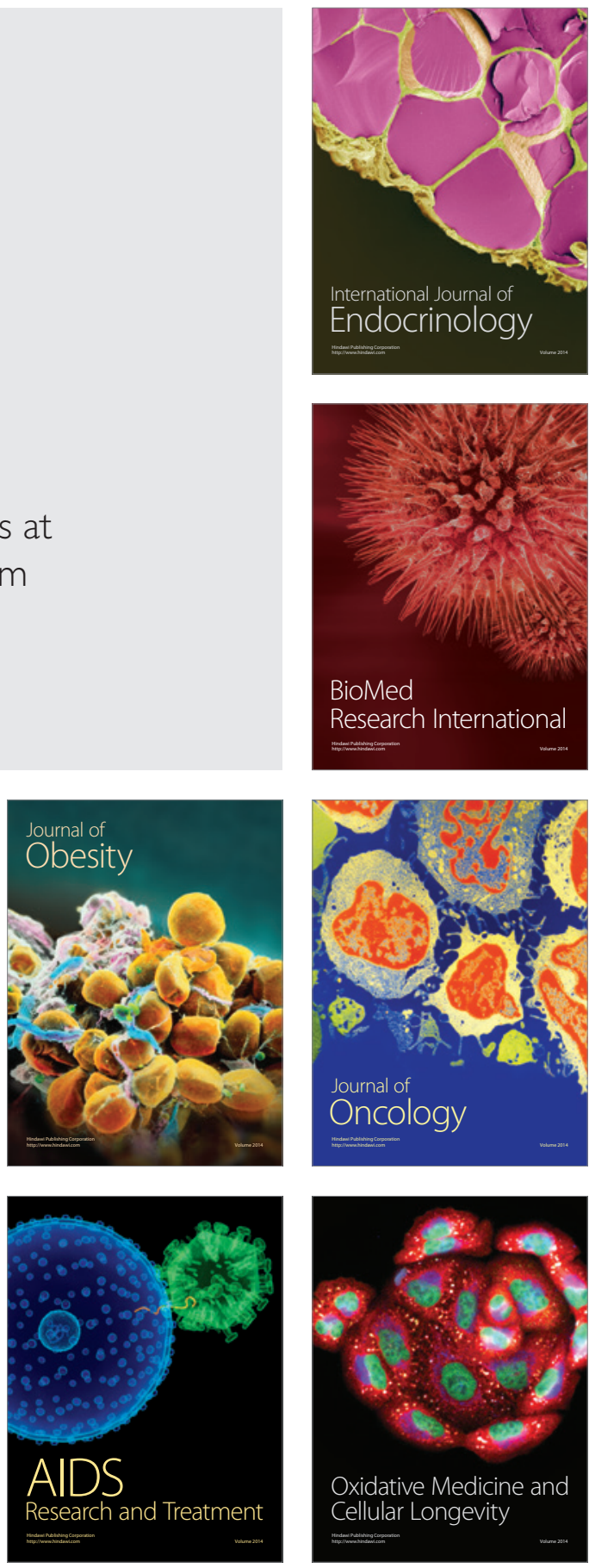